\title{
Search for the light dark matter with an X-ray spectrometer
}

\author{
A. Boyarsky, ${ }^{1,2,3}$ J.-W. den Herder, ${ }^{4}$ A. Neronov, ${ }^{5}$ and O. Ruchayskiy ${ }^{6}$ \\ ${ }^{1}$ CERN, PH/TH, CH-1211 Geneve 23, Switzerland \\ ${ }^{2}$ École Polytechnique Fédérale de Lausanne, Institute of Theoretical Physics, \\ FSB/ITP/LPPC, BSP 720, CH-1015, Lausanne, Switzerland \\ ${ }^{3}$ On leave from Bogolyubov Institute of Theoretical Physics, Kyiv, Ukraine \\ ${ }^{4}$ SRON, The Netherlands Institute for Space Research, \\ Sorbonnelaan 2, 3854 CA Utrecht, the Netherlands \\ ${ }^{5}$ INTEGRAL Science Data Center, Chemin d'Écogia 16, \\ 1290 Versoix, Switzerland and Geneva Observatory, \\ $51 \mathrm{ch}$. des Maillettes, CH-1290 Sauverny, Switzerland \\ ${ }^{6}$ Institut des Hautes Études Scientifiques, Bures-sur-Yvette, F-91440, France
}

\begin{abstract}
Sterile neutrinos with the mass in the $\mathrm{keV}$ range are interesting warm dark matter (WDM) candidates. The restrictions on their parameters (mass and mixing angle) obtained by current Xray missions (XMM-Newton or Chandra) can only be improved by less than an order of magnitude in the near future. Therefore the new strategy of search is needed. We compare the sensitivities of existing and planned X-ray missions for the detection of WDM particles with the mass $\sim 1-20 \mathrm{keV}$. We show that existing technology allows an improvement in sensitivity by a factor of 100 . Namely, two different designs can achieve such an improvement: [A] a spectrometer with the high spectral resolving power of $0.1 \%$, wide (steradian) field of view, with small effective area of about $\mathrm{cm}^{2}$ (which can be achieved without focusing optics) or [B] the same type of spectrometer with a smaller (degree) field of view but with a much larger effective area of $10^{3} \mathrm{~cm}^{2}$ (achieved with the help of focusing optics). To illustrate the use of the "type A" design we present the bounds on parameters of the sterile neutrino obtained from analysis of the data taken by an X-ray microcalorimeter. In spite of the very short exposure time $(100 \mathrm{sec})$ the derived bound is comparable to the one found from long XMM-Newton observation.
\end{abstract}

\section{STERILE NEUTRINO AS WDM CANDIDATE.}

During the last year a number of works appeared, devoted to search for the decay signal of a DM candidate - sterile neutrino - in the X-ray spectra of astrophysical objects $[1,2,3,4,5,6,7,8,9]$. Indeed, it was noticed long ago [10] that a right-handed neutrino with its mass in the keV range presents a viable warm dark matter (WDM) candidate. Such a particle would possess a specific radiative decay channel and therefore one can search for its decay line in the X-ray spectra of astrophysical objects [11, 12].

The recent spark of interest in the search for sterile neutrino DM has several reasons. First, for the direct search of a DM particle, a particle physics model is needed. Most of the particle physics candidates (axion, supersymmetric particles, etc.) would constitute cold dark matter (CDM). CDM models have several difficulties which could be resolved by a warm DM with the particle mass in the keV range. In particular, WDM can ease the problem of the dark halo structures in comparison with the CDM scenario [13, 14, 15]. Second, as the Standard Model of particle physics (SM) does not contain a DM candidate, most of the extensions of the SM ( like, for example, supersymmetry) require to assume the existence of many new particles and/or validity of new fundamental principles. Such extensions are not based on any available experimental data, but on theoretical arguments only. From this point of view, the extension of the
SM with several right-handed neutrinos (i) is, maybe, a minimal extension of the SM one can imagine; (ii) is based on experimental data; (iii) provides naturally a warm DM candidate.

Indeed, the existence of right-handed neutrino particles would provide the most natural explanation of neutrino oscillations, which cannot be explained within the Standard Model (see e.g. [16] for a review of neutrino oscillations). Adding three right-handed (or sterile) neutrinos, neutral with respect to all the Standard Model (SM) gauge interactions, makes the fermion sector of the SM fully symmetric, as every left-handed fermion obtains a right-handed counterpart. It has been demonstrated recently in Refs. [17, 18] that the parameters of these right-handed particles can be chosen in such a way that this model resolves another problem of the SM explains the excess of baryons over antibaryons in the Universe (the baryon asymmetry). At the same time it does not spoil the predictions of the Big Bang nucleosynthesis and accommodates the data on neutrino oscillations. For this to be true, the masses of the two of these sterile neutrinos should be chosen in the range $300 \mathrm{MeV} \lesssim M_{2,3} \lesssim 20 \mathrm{GeV}$, while the mass of the third (lighter) sterile neutrino is arbitrary (as long as it is below $\left.M_{2,3}\right)$. As the coupling of this lightest right-handed particle with ordinary matter can be made arbitrary weak (for example, it can entirely decouple of the other SM particles, while being produced in the early Universe via the decay of the inflaton [19]), this particle provides a viable DM candidate. For this its mass should satisfy the universal Tremaine-Gunn lower bound [20]: 
$M_{\mathrm{DM}} \gtrsim 300-500 \mathrm{eV}$. In particular, its mass can be in $\mathrm{keV}$ range.

For other interesting applications of sterile neutrinos with the mass in the keV range in astrophysics and cosmology see [21, 22, 23, 24, 25] and references therein.

The details of the process of structure formation in the Universe depend on the mass of DM particles. In principle, comparison of the results of numerical modeling of structure formation with Lyman- $\alpha$ forest data allows to obtain a lower bound on the DM particle mass $[26,27]$. However, it turns out that such a bound is model-dependent [27, 28]. This means that experimentally interesting energy range for the search of the DM decay line is anywhere above the lower limit determined by the Tremaine-Gunn bound, $E \gtrsim 150-250 \mathrm{eV}$.

If the mass of the sterile neutrino is less than the electron rest mass, it can decay into a photon and an active neutrino [29]. ${ }^{1}$ As the mass of the active neutrino is much smaller than $1 \mathrm{keV}$, the photon is essentially monoenergetic: $E_{\gamma}=\frac{M_{s}}{2}$. The radiative decay width $\Gamma$ is traditionally parameterized in terms of mass $M_{s}$ and mixing angle $\theta$ - measure of the interaction of the sterile neutrino with its active counterparts. The radiative decay width is expressed via $M_{s}$ and $\theta$ as [29, 36]:

$$
\begin{aligned}
\Gamma & =\frac{9 \alpha G_{F}^{2}}{1024 \pi^{4}} \sin ^{2} 2 \theta M_{s}^{5} \\
& \simeq 1.38 \times 10^{-22} \sin ^{2}(2 \theta)\left[\frac{M_{s}}{\mathrm{keV}}\right]^{5} \sec ^{-1} .
\end{aligned}
$$

where $\alpha$ is the fine-structure constant and $G_{F}=1.166 \times$ $10^{-5} \mathrm{GeV}^{-2}$ is the Fermi coupling constant. The photon flux from the DM decay is given by

$$
F_{\mathrm{DM}}=\frac{E_{\gamma}}{M_{s}} \Gamma \int_{\text {fov cone }} \frac{\rho_{\mathrm{DM}}(\vec{r})}{4 \pi\left|\vec{D}_{L}+\vec{r}\right|^{2}} d^{3} \vec{r} .
$$

Here $\vec{D}_{L}$ is the distance between the observer and the center of the observed object, $\rho_{\mathrm{DM}}(r)$ is the DM density and the integration is over the DM distribution inside the (truncated) cone - solid angle, spanned by the field of view (FoV) of a telescope. If the observed object is far $^{2}$, Eq. (2) simplifies to:

$$
F_{\mathrm{DM}}=\frac{M_{\mathrm{DM}}^{\mathrm{fov}} \Gamma}{4 \pi D_{L}^{2}} \frac{E_{\gamma}}{M_{s}}
$$

\footnotetext{
1 Although throughout this paper we are talking mostly about the sterile neutrino, all the results can be applied to any DM particle, possessing the monoenergetic radiative decay channel, emitting photon of energy $E_{\gamma}$ and having decay width $\Gamma$. For earlier works, discussing cosmological and astrophysical effects of decaying DM see $[30,31,32,33,34]$. The extensive review of the results can be also in the book [35].

${ }^{2}$ Namely, if luminosity distance $D_{L}$ is much greater than the characteristic scale of the DM distribution $\rho_{\mathrm{DM}}(r)$.
}

where $M_{\mathrm{DM}}^{\text {fov }}$ is the mass of DM within a telescope's field of view (FoV).

Clustering of the DM at small red shifts results in the enhancement of the DM decay signal in the direction of large nearby mass concentrations, such as the Milky Way halo, nearby galaxies and galaxy clusters. Characterizing the clustering scale through the typical overdensity compared to the mean cosmological DM density $\rho_{\mathrm{DM}}^{0}$

$$
\mathcal{R}=\rho / \rho_{\mathrm{DM}}^{0}
$$

$\left(\mathcal{R} \sim 10^{6}\right.$ for a galaxy, $\mathcal{R} \sim 10^{3}$ for a galaxy cluster $)$ and through its size, $D \sim 10 \mathrm{kpc} \sim 10^{-5} H_{0}^{-1}\left(H_{0}\right.$ is the Hubble constant) for a galaxy, $D \sim 1 \mathrm{Mpc} \sim 10^{-3} H_{0}^{-1}$ for a galaxy cluster, one can find that the DM decay flux from a particular overdensity is comparable to the background DM decay signal. Indeed, in Eq.(3) $M_{\mathrm{DM}}^{\text {fov }} \simeq$ $\mathcal{R} \rho_{\mathrm{DM}}^{0} D D_{\theta}^{2} \Omega_{\mathrm{fov}}$ and therefore

$$
\frac{F_{\mathcal{R}}}{F_{\mathrm{XRB}}} \sim \mathcal{R} D H_{0} \sim 1
$$

However, the spectra of the DM signal from the background and from a galaxy or galaxy cluster are different. The flux from a nearby object would be detected as a Doppler-broadened line of the width

$$
\Delta E_{\text {line }}=\frac{v_{\text {vir }}}{c} E_{\gamma}
$$

where $v_{\text {vir }}$ is the virial velocity of the DM particles $\left(v_{\text {vir }} \sim\right.$ $10^{-2} c$ for a galaxy cluster, $v_{v i r} \sim 10^{-3} c$ for a galaxy). At the same time, the DM decay contribution into XRB is produced by the decays at red shifts $z \sim 0 \div 1$ and, as a result the DM decay line is broadened to $\Delta E \sim M_{s} / 2$. Thus, in spite of the fact that the compact DM sources at $z \simeq 0$ give just moderate enhancement of the DM decay flux, the enhancement of the signal in the narrow energy band centered on the line energy $E=M_{s} / 2$ can be large for the instruments with high spectral resolution.

\section{SENSITIVITY OF X-RAY TELESCOPES FOR DM DETECTION.}

As it is discussed in the previous Section several types of astrophysical objects are expected to produce comparable strength DM decay fluxes. Galaxy clusters and nearby dwarf galaxies are extended sources of the size of about $1^{\circ}$. The Milky Way halo is expected to produce a diffuse signal detectable from all directions.

Most of the currently operating X-ray telescopes are not optimized for the study of diffuse emission and/or very extended sources. For example, the field of view of Chandra and XMM-Newton are, respectively, $256 \operatorname{arcmin}^{2}$ and $700 \operatorname{arcmin}^{2}$, much smaller than the angular size of the typical DM dominated sources.

To improve the existing bounds on DM parameters $[1,2,3,4,5,6,7,8,9]$ using these instruments, one can look for the sources with optimized "signal-to-noise" 


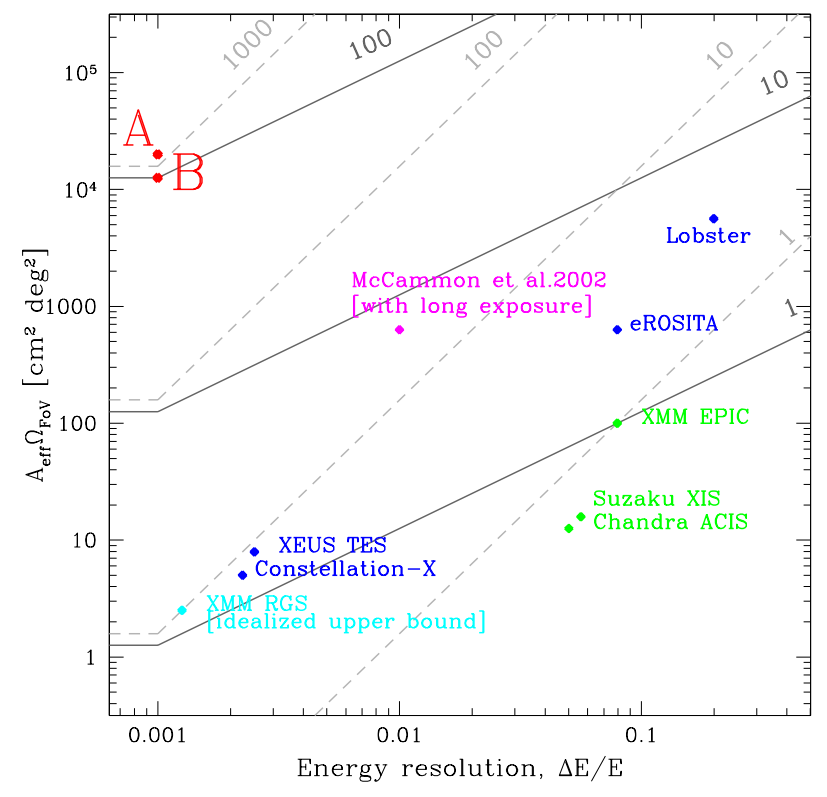

FIG. 1: Comparison of sensitivities of existing and proposed/planned X-ray missions for the detection of the DM decay line from the Milky Way DM halo. The plot shows the characteristics of different telescopes in the two parameter space relevant for the diffuse DM line detection: "Energy resolution" vs. "Grasp". The sensitivity of XMM-Newton is taken as a reference. Solid lines limit from below the regions of parameter space in which the improvement of sensitivity by factors of 1,100 and 100 can be achieved (each line is marked by the corresponding numerical factor). Dashed lines show the improvement of conservative "total flux" bounds on neutrino mixing angle (see text for explanations) in the case of non-detection of the DM decay line.

ratio (as e.g. dwarf galaxies [3]) and study them with prolonged observations. However, as we have discussed in the previous section, the expected DM signal does not vary too much from object to object. Even if an object is undetected in X-rays, the bounds are defined by statistical error which behaves as $\sqrt{t_{\exp }}$. Therefore, improving the results by an order of magnitude requires an exposure time two orders of magnitude longer. Thus, further improvement of the results will be very slow and would require a lot of observational time of existing satellites.

A qualitative improvement of the bounds on the DM parameters (or final detection of the DM decay signal) can be achieved only with a qualitatively new instrumentation. To find what experimental set up can be considered as "optimized" for the DM search, it is useful to compare the sensitivities of existing and planned X-ray missions for the detection of the DM decay signal.

To this end one needs to distinguish two possible situations - strong line and weak line regimes. If the line is weak (i.e. there is no line detectable against the continuum at more than several $\sigma$ ) and the background signal can be fitted well by a convincing physical model (or just a featureless power law model), detectabilty of the line is roughly defined by the statistical error of the background flux in the corresponding energy bin. As the background count rate in a narrow energy band, centered at the line energy, is proportional to the spectral resolution of the instrument $\Delta E$, the significance of line detection scales as the inverse of the square root of spectral resolution

$$
\text { LINE SIGNIFICANCE } \sim \frac{1}{\sqrt{1+\Delta E / E W}} .
$$

For a given total flux in the line, the sensitivity for the line detection improves as

$$
\text { SENSITIVITY } \sim \frac{1}{\sqrt{\Delta E}}
$$

until the spectral resolution reaches the equivalent width (EW) of the line. In this case one reaches the "strong line" regime in which the detection significance does not depend on the statistical error of background anymore. The relation (8) holds until the spectral resolution becomes so good that it either reaches the intrinsic width of the line (6) (that is, $\Delta E \sim 10^{-2} E$ for galaxy clusters and $\Delta E \sim 10^{-3} E$ for galaxies) or the background count rate in the narrow energy bin becomes so small that one looks for a line signal in a background-free regime.

If the line is strong the sensitivity is defined by the intensity of the line itself. From this point of view, making $\Delta E$ smaller could not just increase the sensitivity of an instrument, but change the situation from the weak line to the strong line regime and, therefore, increase the sensitivity much more significantly. The condition for the line flux to exceed that of the background scales as $1 / \Delta E$.

Additionally, the search of the DM decay line in the Xray energy band is complicated by the fact that this line can be easily confused with atomic emission lines present in the emission spectra of astrophysical plasmas. Uncertainties of the models of diffuse emission from the warm and hot plasma in the Galaxy prevent a proper statistical analysis of the data. This difficulty is the main reason why many works, deriving the bounds on the DM sterile neutrino parameters, consider a simpler approach which gives weaker, but more robust constraints. Namely, one simply requires that the DM decay line flux in a given narrow energy band does not exceed the total background flux in the same energy bin (c.f. [3, 4, 5, 6, 7]). Although such approach does not allow to detect a line, if it is present in the data, it permits to derive a "background model independent" bounds.

As explained above, within the "total flux restrictions" approach, the bounds on the neutrino parameters, derived from the data, improve as

$$
\text { TOTAL FLUX BOUND } \sim \frac{1}{\Delta E}
$$

rather than as $1 / \sqrt{\Delta E}$, as in the statistical analysis approach. In Fig. 1 we show with dashed grey lines the 
improvements of the upper bound on the DM sterile neutrino mixing angle which can be achieved within the "total flux restrictions" approach. One can see that e.g. for an instrument with the spectral resolving power of about $10^{3}$ and collecting power of $10^{4} \mathrm{~cm}^{2} \mathrm{deg}^{2}$ the improvement can by by 3 orders of magnitude, compared to the bounds derived from XMM-Newton data.

The imaging instruments on existing satellites have spectral resolution of about $10 \%$. However, there are grating spectrometra on board of both XMM-Newton and Chandra telescopes. The resolving power of Reflection Grating Spectrometer (RGS) on board of XMMNewton is 10-40 times better than that of EPIC cameras. Even if it were possible to use the full field of view of this instrument with its maximal spectral resolution, this would provide an order of magnitude improvement of sensitivity, as compared to the EPIC camera of XMMNewton. Such a spectral resolution is possible, however, only for point sources. For spatially extended objects the resolution degrades proportionally to the angular size of the source (see e.g. [37, 38]). Taking into account that for the DM line search one deals with extended sources (with the angular size larger than that of the field of view of the RGS), one finds that the spectral resolution of grating instrument in this case is even worse than that of EPIC camera. To recover a better spectral resolution for an extended object, one must use modeling, using a surface brightness profile of a given object. For the DM search, however, it should be noticed that the surface brightness profile for the DM is much flatter than the surface brightness for the intracluster or intergalactic media, as it is proportional not to the square of the density profile, but to the density profile itself (as we are looking for the one-particle decay process). This makes the effective size of the source larger and should be taken into account in the corresponding data processing. As a result, the existing gratings of XMM and Chandra can hardly improve the sensitivity for the DM search significantly.

Microcalorimeters provide an alternative to the gratings in achieving high spectral resolution. The existing technology (c.f. e.g. EURECA - EURopean-JapanEse Calorimeter Array [39, 40, 41]) allows to construct an Xray detector with $0.1 \%$ spectral resolution at several $\mathrm{keV}$ energies.

An obvious requirement for any instrument aimed at high-resolution spectroscopic study is the maximal possible effective collecting area, $A_{\text {eff }}$, needed to increase the photon statistics in the narrow energy bands corresponding to the spectral lines. The statistics of the background signal also grows with $A_{\text {eff }}$ which leads to the fact that the sensitivity scales only proportionally to the square root of the area

$$
\text { SENSITIVITY } \sim \sqrt{A_{\text {eff }}}
$$

The need to maximize the effective area has pushed the design studies of the planned X-ray telescopes to consider the multi-spacecraft configuration in which a mirror with a large collecting area $\left(5 \mathrm{~m}^{2}\right.$ in the case of $\left.X E U S\right)$ flies separately from the detector.

However, if one is interested in the search of DM line from the Milky Way halo, a simpler design allows to achieve sensitivity higher than the one that would be reached with $X E U S$. The point is that one can increase the photon statistics by increasing the field of view of the telescope (even the "wide field" camera on board of $X E U S$ is supposed to have a tiny field of view of just 6 arcmin). For the case of the MW halo the line and background photon statistics are just proportional to the size of the FoV, $\Omega_{\text {fov }}$, which means that the sensitivity for the DM line detection scales as

$$
\text { SENSITIVITY } \sim \sqrt{\Omega_{\mathrm{fov}}}
$$

We compare the sensitivities of existing and future missions for the detection of the DM decay line signal from the Milky Way halo on the FIG. 1, taking sensitivity of XMM-Newton EPIC camera as the reference. The resolving power is plotted along the $\mathrm{X}$-axis of FIG. 1. Since the overall photon statistics is proportional to the product $A_{\text {eff }} \Omega_{\text {fov }}$ it is convenient to range the X-ray missions according to their "grasp", $A_{\text {eff }} \Omega_{\text {fov }}$. This parameter is plotted along the $\mathrm{Y}$ axis in FIG. 1. The diagonal solid lines, marked by the corresponding numbers, show the relative improvement in sensitivity for different missions, as compared to XMM-Newton. The turnover of the lines at the resolving power $\Delta E / E \sim 10^{-3}$ is related to the fact that further improvement of the spectral resolution will not lead to the improvement of sensitivity, because one hits the natural width of the line, determined by the Doppler broadening due to the random motions of the DM particles in the gravitational potential of the Galaxy. In Fig. 1 the "XMM RGS" point (dark green dot) marks the idealized upper bound for the grating if one could have used its full field of view with maximal spectral resolution. As discussed above, to use gratings in case of extended objects, one needs to model surface brightness profile of both DM and gas components. As one sees from FIG. 1, even this, hard to achieve, setup gives at most moderate improvement compared to $X M M$ Newton/EPIC. Shown in magenta color is the spectrometer used by McCammon et al. [39] (see Section IV below). This spectrometer flew on a sounding rocket and therefore had very short exposure time ( $100 \mathrm{sec})$. Plotted on FIG. 1 is the sensitivity of the corresponding device if it were placed on a satellite (in which case it would have much longer exposure). This explains, why the results of Section IV below do not provide an order of magnitude improvement, compared to the XMM-Newton data (c.f. FIG. 5).

Fig. 2 shows a comparison of sensitivities of existing and planned missions for the detection of DM decay line from the nearby dwarf galaxies (we take as a reference a galaxy of the angular size of $\sim 2^{\circ}$ ). The main difference with the case of DM decay line from the Milky Way halo is that the objects have finite angular extent. This means that extending the field of view of an instrument improves the sensitivity only when the size of the field of view is 


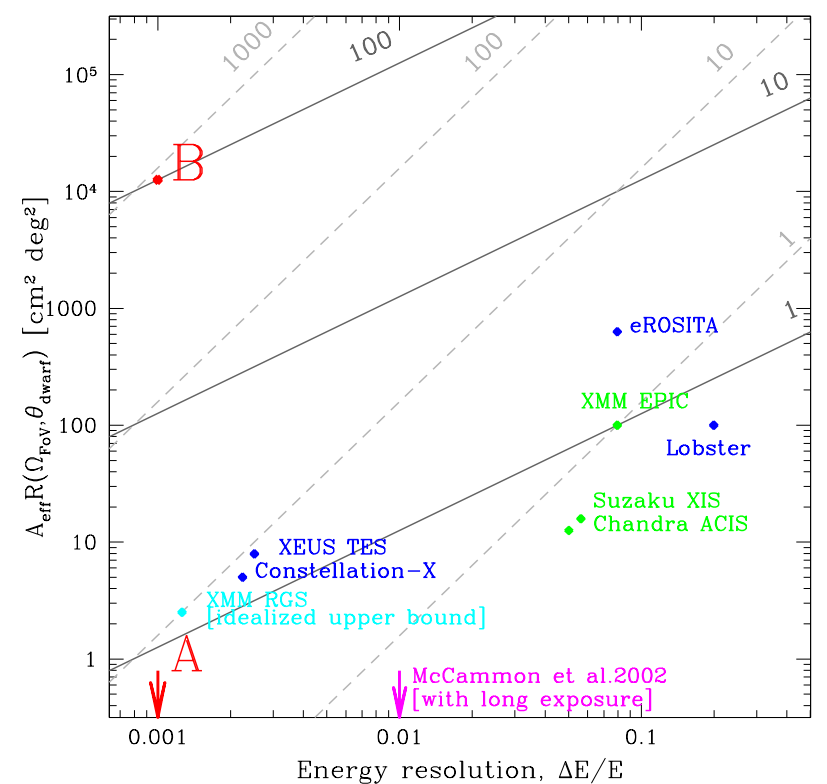

FIG. 2: Same as in Fig. 1, but for the sensitivity for the detection of DM decay line from a nearby dwarf galaxy of the angular size of 2 degrees.

less or equal the angular size of the dwarf galaxy, $\theta_{\text {dwarf }}$. The $Y$ axis of Fig. 2 shows, therefore the product of effective area of an instrument on a function

$$
R\left(\Omega_{\mathrm{FoV}}, \theta_{\mathrm{dwarf}}\right)= \begin{cases}\Omega_{\mathrm{FoV}}, & \text { if } \Omega_{\mathrm{FoV}} \leq \pi \theta_{\mathrm{dwarf}}^{2} \\ \pi \theta_{\mathrm{dwarf}}^{2}, & \text { if } \Omega_{\mathrm{FoV}}>\pi \theta_{\mathrm{dwarf}}^{2}\end{cases}
$$

Another main difference between Fig. 2 and Fig. 1 is that the lines representing the improvement of sensitivity by a factor of 10 and 100 do not have a turnover at $\Delta E / E \simeq 10^{-3}$, because the velocity dispersion of particles moving in the halo of dwarf galaxy is an order of magnitude less than the velocity dispersion of DM particles in the Milky Way halo. Thus, the Doppler broadening of the line is not observable until the spectral resolution becomes $\Delta E / E \sim 10^{-4}$.

Finally, Fig. 3 shows comparison of sensitivities of different missions for the detection of the DM decay line from galaxy clusters. One can see that because of the larger velocity dispersion of DM particles in the galaxy clusters the improvement of the spectral resolution does not lead anymore to the improvement of sensitivity already when the resolution $\Delta E / E \sim 1 \%$ is reached. Even disregarding the fact that the detection of DM decay line from the galaxy clusters is complicated by the fact that clusters are strong sources of continuum and atomic line X-ray emission, one can see from Fig. 1 that no significant improvement of the bounds imposed by $X M M$ Newton observations [2] can be achieved with and of existing and proposed experiments.



FIG. 3: Same as in Fig. 1, but for the sensitivity for the detection of DM decay line from a galaxy cluster of the angular size of 2 degrees.

\section{OPTIMAL EXPERIMENTAL SET-UP.}

From Figs. 1, 2 one can clearly see what qualitative improvements of experimental set up are needed to improve sensitivity for the DM decay line detection. If one aims at the detection of the DM decay line from the Milky Way halo, one can choose two different ways for such improvements, which we describe as "type A" and "type B" designs below.

First of all, it is clear that an improvement of spectral resolution down to the theoretical "ultimate" resolution of $\Delta E / E \sim 0.1 \%$ enables to improve the sensitivity by a factor of 10 compared to the sensitivity of XMM-Newton. A microcalorimeter-type detector can achieve this spectral resolution. Further improvements of the sensitivity can be achieved by increasing the "grasp" of the instrument.

In the "type A" design, one maximizes the "grasp" by increasing the size of the field of view up to (several) steradian. The wide field of view of the instrument makes the use of focusing optics impossible and the only way to maximize the effective area is to increase as much as possible the geometrical area of the detector. In Fig. 1 we tentatively assume that an area of $3 \mathrm{~cm}^{2}$ (10 times larger than the detector of McCammon et al. [39]) is reachable for a microcalorimeter-type detector (although this can be challenging task with an existing technology).

In the "type B" design one maximizes the "grasp" by increasing the effective collection area of the detector with the help of the imaging optics. In this case moderately extending the field of view to $1.5^{\circ} \times 1.5^{\circ}$, 
and increasing the effective collection area to $1000 \mathrm{~cm}^{2}$ will enable to improve the XMM-Newton total flux restrictions by three orders of magnitude and to increase the sensitivity for the line detection by more than an order of magnitude, provided that a microcalorimeter type detector is installed in the focal plane.

One should note that both types of designs have their limitations. For example, the "type A" set-up will be difficult to use at the energies below $1 \mathrm{keV}$ because the DM decay signal will be contaminated by the strong foreground emission from the local hot bubble, which contains a huge amount of atomic emission lines. In this case the lack of proper imaging capabilities would make the disentanglement of the DM decay line signal from the forest of atomic lines extremely difficult. On the other side, in the "type B" set-up the use of the focusing optics essentially limits the size of the field of view, especially at the energies above $2 \mathrm{keV}$ (higher energies require longer focal length and hence larger detector area). Thus at the energies above several keV "type B" design quickly becomes technically not feasible with the current day technology.

Apart from the DM search the wide field of view both types of design can serve for spectrometer for several engineering and scientific tasks. A focusing X-ray telescope with a $1.5^{\circ}$ field of view and excellent spectral resolution ("type B" design) can serve for a variety of astrophysical problems. The flight of the wide field of view spectrometer ("type A" design) can be considered as a test of the X-ray spectrometers of the next generation "big" Xray mission, such as XEUS or Constellation X. A slightly modified version of the "bare spectrometer" design would also make soft X-ray measurements of the prompt emission from gamma-ray bursts (GRBs). This is a particularly interesting task in the view of the claimed detection of transient line and/or edge-like features in the X-ray spectra of prompt emission of several GRBs [42, 43].

In fact, none of the proposed X-ray mission is optimized to verify this claim, because the detection of Xray lines and edges in the spectra of prompt emission of GRBs requires a wide field of view X-ray detector. Up to now only the Wide Field Camera (WFC) on board of BeppoSAX mission satisfied this requirement (the field of view of about $40^{\circ}$ and moderate spectral resolution of $\sim 20 \%$ ). The WFC has detected the spectral features (probably associated with iron) in 5 GRBs [42, 43]. Sensitivity of the wide field of view spectrometer for the line detection will be comparable to the sensitivity of the WFC, because the decrease of the effective area (by a factor of 20) will be compensated by the gain in the spectral resolution by a factor of 200 .

Although the "type A" and "type B" designs have comparable sensitivity for the detection of the DM decay line from the Milky Way halo, the sensitivity of the wide field of view design for the detection of DM decay signal from the nearby dwarf galaxies is extremely low, as one can see from Fig. 2. However, this does not make the "type B" design preferable compared to the "type A" one. Indeed, as it is mentioned above, the "type B" instruments can operate only in a narrow energy range $0.1-($ several $) \mathrm{keV}$, while the energy region interesting for the sterile neutrino DM search extends definitely above 1-2 keV energies. At higher energies the wide field of view spectrometer becomes the only available configuration which will provide an increase of sensitivity by a factor of 100 compared to the sensitivities of existing instruments.

\section{ANALYSIS OF THE X-RAY SPECTROMETER DATA.}

A "prototype" of a cryogenic X-ray spectrometer has already been successfully tested in flight [39]. The detector was composed of 36 micro-calorimeters of the surface area of $1 \mathrm{~mm}^{2}$ each and had a wide, 1 sr field of view. In this Section we will show that, although the flight time of this calorimeter was short, about 100 seconds, good characteristics of the detector (spectral resolution of $10 \mathrm{eV}$ ) allow this instrument to compete with XMM-Newton in restricting parameters of sterile neutrino DM.

We have analyzed the data obtained by the X-ray spectrometer ${ }^{3}$ in order to derive the upper bounds on the neutrino mixing angle as a function of the mass. ${ }^{4}$ At the energies below $\mathrm{keV}$ the signal collected by the detector is dominated by the diffuse X-ray background of which some fraction is produced by distant active galactic nuclei $[44,45,46]$ and can be modeled with an absorbed power-law, while the rest is due to the thermal emission from the local hot bubble (the temperature $T \simeq 0.1 \mathrm{keV}$ ). The thermal emission is dominated by the atomic emission lines. The X-ray background spectrum measured by the X-ray spectrometer and the identifications of the detected atomic lines can be found in Ref. [39].

The presence of bright emission lines in the background spectrum complicates the search of the DM decay line. The main problem is that the DM can "hide" behind an atomic line if the energies of two lines are close enough. The problem of "hiding behind the line" can, in principle, be relaxed if one properly models the background thermal emission: the ratios of the intensities of the multiple emission lines from the same element are fixed if the temperature of the gas is known. The DM decay line would then reveal itself if an "anomalous" ratio between line intensities is found at a particular energy. In the statistical analysis of the data, addition of the DM decay line at the right energy on top of the thermal emission model would then improve the quality of the model fit to

\footnotetext{
${ }^{3}$ We thank Prof. D. McCammon for providing us the data for the analysis

${ }^{4}$ When this work was finished, the paper [9] appeared, which compared estimates for the neutrino line flux in the DodelsonWidrow model at $E=1 \mathrm{keV}$ with the flux, measured by [39] at the same energy.
} 


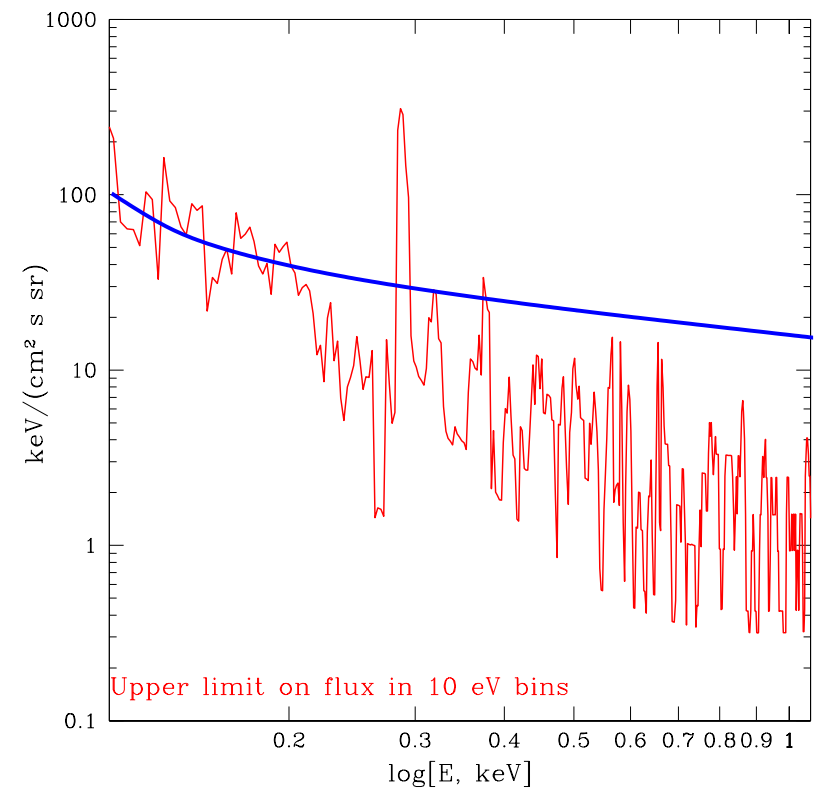

FIG. 4: Red thin line: an upper limit on the flux in the dark matter decay line as a function of energy. Blue thick line: average level (line features smoothed) of soft X-ray spectrum in the $0.1-1 \mathrm{keV}$ energy range.

the data by removing the "anomaly" in the line intensity ratio.

The statistical analysis outlined above has sense at large enough signal to noise ratios. However, because of the short exposure time of the X-ray spectrometer under consideration, the brightest lines were detected at $3-4 \sigma$ level and statistical analysis gives the results comparable to the results obtained with a more simple analysis method which does not depend on the assumptions about the background emission model.

The background model independent bound on the neutrino parameters can be derived from the fact that the flux in a narrow line at a given energy can not exceed the total detected flux (possible line plus background) in a narrow energy band of the width equal to the energy resolution of the instrument and centered at the line energy. We have "scanned" the whole energy interval of interest, $0.2 \mathrm{keV}<E<1 \mathrm{keV}$, calculating the maximal allowed flux at $3 \sigma$ level in a narrow energy window of the width $10 \mathrm{eV}$, equal to the energy resolution of the spectrometer. With such a method one does not have a possibility to really detect a DM decay line, one can only obtain a robust restriction on the DM parameters.

The limit on the total flux in $10 \mathrm{eV}$ energy bins as a function of energy is shown in FIG. 4 with the red solid line. One can see that the upper limit on the total flux is weaker at the energies where real atomic lines are present. The X-ray photon flux is, in general, affected by the photo-electric absorption in the interstellar medium of the Galaxy. In order to correct the data for the effect

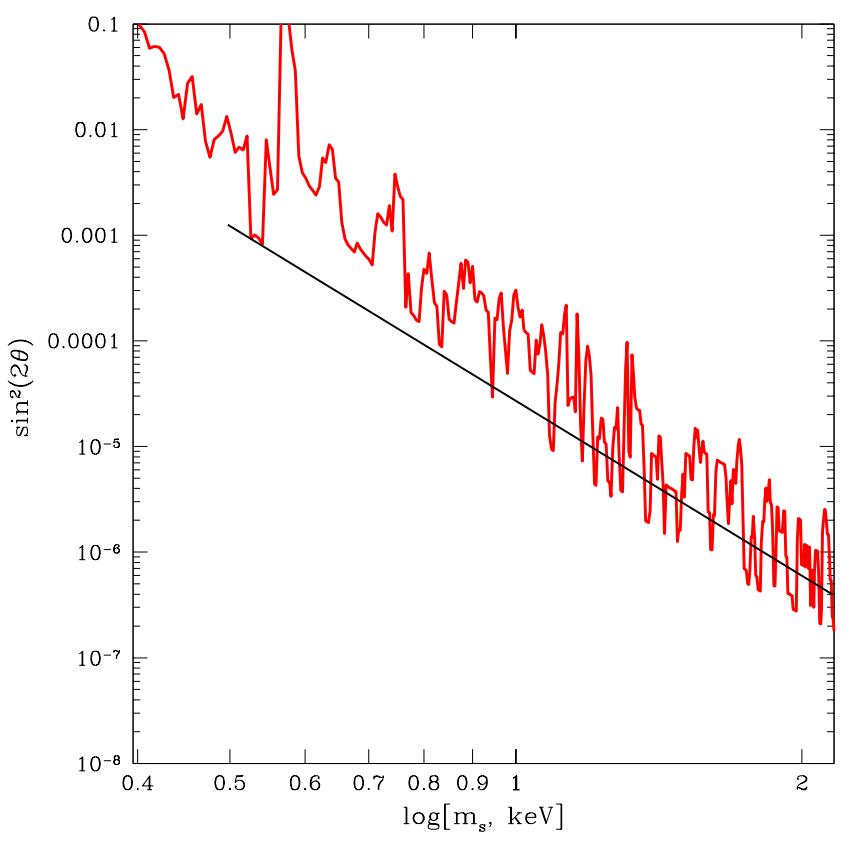

FIG. 5: Exclusion plot in the $\left(m_{s}, \sin ^{2} 2 \theta\right)$ parameter space obtained from the analysis of the X-ray spectrometer data of McCammon et al. [39] (solid thick red line). For comparison, the upper limit on the mixing angle obtained from the XMM-Newton observation of the Large Magellanic Cloud obtained in [3] is shown by the solid thin black line.

of photoelectric absorption, one has to know the hydrogen column density in the direction of the source. In the case of interest, the source (the DM particles decaying in the Galactic halo) is distributed all over the Galaxy and a correct calculation of the effect of absorption should involve an integration of absorption of the DM signal along the line of sight. The result of such a calculation would depend on the assumptions about the model of spatial distribution of the hydrogen in the Galaxy. In order to impose a conservative upper limit on the possible DM line flux one can use a simplified procedure which slightly over-estimates the effect of photo-electric absorption, namely, one can assume that the signal is absorbed on the total Galactic hydrogen column density $N_{H}(l, b)$ in a given direction. Using the known distribution of the hydrogen column density [47], we find that the mean hydrogen column density throughout the spectrometer field of view in the pointing direction of the instrument $\left(l=+90^{\circ}, b=+60^{\circ}\right.$ in Galactic coordinates $)$ is $N_{H} \simeq 1.5 \times 10^{20} \mathrm{~cm}^{-2}$. FIG. 4 shows the data corrected for the absorption on this hydrogen column density.

In order to derive the upper bound on the neutrino mixing angle as a function of the neutrino mass from the above upper limit on the DM decay line flux, one has to calculate the expected DM decay line flux within the $1 \mathrm{sr}$ field of view of the detector. The field of view of the detector was pointed in the direction $l=+90^{\circ}, b=+60^{\circ}$ in Galactic coordinates. The expected DM decay line flux 
(Eq. (2)) in the case of Galactic DM halo was computed in [3]. It varies from its minimum value, $F_{\mathrm{DM}, \text { min }}$ from the direction of Galactic anti-center to the maximum, $F_{\mathrm{DM}, \max } \simeq 6 F_{\mathrm{DM}, \min }$ from the direction of the Galactic center. For a conservative upper limit we can assume that the flux from the direction of the North Galactic Pole is about the minimal flux $F_{\mathrm{DM}, \min }$. Relating via Eq. (1) the DM decay width $\Gamma$ to parameters of sterile neutrino DM $M_{s}$ and $\sin ^{2}(2 \theta)$ we obtain:

$$
F_{\mathrm{DM}}=3.84 \times 10^{4} \sin ^{2}(2 \theta)\left[\frac{M_{s}}{\mathrm{keV}}\right]^{5} \frac{\mathrm{keV}}{\mathrm{cm}^{2} \cdot \mathrm{sec} \cdot \mathrm{sr}}
$$

The emitted photon has the energy $E_{\gamma}=M_{s} / 2$. The bound on the neutrino mixing angle as a function of neutrino mass derived from the upper limit on the flux in $10 \mathrm{eV}$ energy bins (FIG. 4) is shown in Figure 5. For comparison we show in the same figure the bound derived from the statistical analysis of the XMM-Newton data [3]. A remarkable fact is that in spite of the very short exposure time (100 s compared to $18 \mathrm{ksec}$ exposure in XMM-Newton LMC data) the bounds derived from the data of the two instruments are comparable. (Moreover, the bound from the X-ray spectrometer is even gets better than that of XMM-Newton for some DM masses $M_{s} \geq 1 \mathrm{keV}$ (i.e with the line energy above $0.5 \mathrm{keV}$ )). This demonstrates the advantages of the "type A" experimental set-up, outlined above: the large field of view makes the "grasp" of an instrument even with a small effective area (like the spectrometer under consideration) comparable to the "grasp" of the focusing instrument with a small field of view (like XMM-Newton). Combined with a good spectral resolving power, such design turns out to be better suited for the DM line search than XMM- Newton.

\section{CONCLUSION}

In this paper we analyzed existing and planned X-ray missions with respect to their ability to detect signal of radiatively decaying DM. Our analysis shows that two types of experimental setups enable to improve the sensitivity for the detection of the DM decay line by a factor of 100 compared to the existing instruments, such as XMM-Newton and Chandra. Namely, a high spectral resolution detector with resolving power $\Delta E / E \sim 10^{3}$ incorporated either in a wide (steradian scale) field of view telescope with limited imaging capabilities ("type A" design) or into a focusing telescope with much smaller (degree scale) field of view, but with a much larger collection area ("type B design") can achieve such an improvement. Both type A and type B designs are optimized for the search of the DM decay signal from the DM halo of the Milky Way galaxy. The "type B" design will also be able to search the DM decay signal from the nearby dwarf galaxies and from the galaxy clusters. The two designs are complimentary to each other because the "type B" design is optimized for the search of DM signal in the energy range from $0.2 \mathrm{keV}$ up to $1-2 \mathrm{keV}$, while the "type A" design is more suitable for the search of DM signal at the energies above $1 \mathrm{keV}$ (and can be extended to $10-15 \mathrm{keV})$.

\section{Acknowledgements}

We would like to thank D. McCammon for sharing the data with us and T. Maccarone, M. Markevitch, M. Shaposhnikov, I. Tkachev and J. M. in 't Zand for stimulating scientific discussions and for providing very useful comments on the manuscript. The work of A.B. was (partially) supported by the EU 6th Framework Marie Curie Research and Training network "UniverseNet" (MRTNCT-2006-035863). The work of O.R. was supported in part by the European Research Training Network contract 005104 "ForcesUniverse" and by a Marie Curie International Fellowship within the $6^{\text {th }}$ European Community Framework Programme.
[1] A. Boyarsky, A. Neronov, O. Ruchayskiy, and M. Shaposhnikov, MNRAS 370, 213 (2006), astro-ph/0512509.

[2] A. Boyarsky, A. Neronov, O. Ruchayskiy, and M. Shaposhnikov, Phys. Rev. D 74, 103506 (2006), astro$\mathrm{ph} / 0603368$.

[3] A. Boyarsky, A. Neronov, O. Ruchayskiy, M. Shaposhnikov, and I. Tkachev (2006), astro-ph/0603660.

[4] S. Riemer-Sørensen, S. H. Hansen, and K. Pedersen, ApJ 644, L33 (2006), astro-ph/0603661.

[5] C. R. Watson, J. F. Beacom, H. Yuksel, and T. P. Walker, Phys. Rev. D74, 033009 (2006), astro-ph/0605424.

[6] S. Riemer-Sorensen, K. Pedersen, S. H. Hansen, and H. Dahle (2006), astro-ph/0610034.

[7] A. Boyarsky, J. Nevalainen, and O. Ruchayskiy, Submit- ted to A\&A (2006), astro-ph/0610961.

[8] A. Boyarsky, O. Ruchayskiy, and M. Markevitch, Submitted to ApJ. (2006), astro-ph/0611168.

[9] K. N. Abazajian, M. Markevitch, S. M. Koushiappas, and R. C. Hickox, ArXiv Astrophysics e-prints (2006), astro-ph/0611144.

[10] S. Dodelson and L. M. Widrow, Phys. Rev. Lett. 72, 17 (1994), hep-ph/9303287.

[11] A. D. Dolgov and S. H. Hansen, Astropart. Phys. 16, 339 (2002), hep-ph/0009083.

[12] K. Abazajian, G. M. Fuller, and W. H. Tucker, Astrophys. J. 562, 593 (2001), astro-ph/0106002.

[13] P. Bode, J. P. Ostriker, and N. Turok, Astrophys. J. 556, 93 (2001), astro-ph/0010389. 
[14] T. Goerdt, B. Moore, J. I. Read, J. Stadel, and M. Zemp, MNRAS 368, 1073 (2006), astro-ph/0601404.

[15] L. E. Strigari et al. (2006), astro-ph/0603775.

[16] A. Strumia and F. Vissani (2006), hep-ph/0606054.

[17] T. Asaka, S. Blanchet, and M. Shaposhnikov, Phys. Lett. B631, 151 (2005), hep-ph/0503065.

[18] T. Asaka and M. Shaposhnikov, Phys. Lett. B620, 17 (2005), hep-ph/0505013.

[19] M. Shaposhnikov and I. Tkachev, Phys. Lett. B639, 414 (2006), hep-ph/0604236.

[20] S. Tremaine and J. E. Gunn, Phys. Rev. Lett. 42, 407 (1979).

[21] A. Kusenko (2006), hep-ph/0609081.

[22] P. L. Biermann and A. Kusenko, Phys. Rev. Lett. 96, 091301 (2006), astro-ph/0601004.

[23] J. Stasielak, P. L. Biermann, and A. Kusenko (2006), astro-ph/0606435.

[24] A. Kusenko (2006), astro-ph/0609375.

[25] J. Hidaka and G. M. Fuller (2006), astro-ph/0609425.

[26] U. Seljak, A. Makarov, P. McDonald, and H. Trac (2006), astro-ph/0602430.

[27] M. Viel, J. Lesgourgues, M. G. Haehnelt, S. Matarrese, and A. Riotto, Phys. Rev. Lett. 97, 071301 (2006), astroph/0605706.

[28] T. Asaka, A. Kusenko, and M. Shaposhnikov, Phys. Lett. B638, 401 (2006), hep-ph/0602150.

[29] P. B. Pal and L. Wolfenstein, Phys. Rev. D25, 766 (1982).

[30] A. de Rujula and S. L. Glashow, Physical Review Letters 45, 942 (1980).

[31] Z. G. Berezhiani, M. I. Vysotsky, and M. Y. Khlopov, Sov. J. Nucl. Phys. 45, 1065 (1987).

[32] A. G. Doroshkevich, M. I. Khlopov, and A. A. Klypin, MNRAS 239, 923 (1989).

[33] Z. G. Berezhiani, M. I. Vysotsky, V. P. Yurov, A. G. Doroshkevich, and M. Y. Khlopov, Sov. J. Nucl. Phys. 51, 1020 (1990).

[34] Z. G. Berezhiani and M. Y. Khlopov, Sov. J. Nucl. Phys. 52, 60 (1990).
[35] M. Y. Khlopov, Cosmoparticle Physics (World Scientific Pub Co Inc, 1997).

[36] V. D. Barger, R. J. N. Phillips, and S. Sarkar, Phys. Lett. B352, 365 (1995), hep-ph/9503295.

[37] J. R. Peterson, S. M. Kahn, F. B. S. Paerels, J. S. Kaastra, T. Tamura, J. A. M. Bleeker, C. Ferrigno, and J. G. Jernigan, ApJ 590, 207 (2003), astro-ph/0210662.

[38] J. de Plaa, N. Werner, A. M. Bykov, J. S. Kaastra, M. Méndez, J. Vink, J. A. M. Bleeker, M. Bonamente, and J. R. Peterson, A\&A 452, 397 (2006), astro$\mathrm{ph} / 0602582$.

[39] D. McCammon, R. Almy, E. Apodaca, W. Bergmann Tiest, W. Cui, S. Deiker, M. Galeazzi, M. Juda, A. Lesser, T. Mihara, et al., ApJ 576, 188 (2002), astroph/0205012.

[40] P. A. J. de Korte, J. V. Anquita, X. Barcons, P. Bastia, J. Beyer, F. Briones, C. Brockley Blatt, M. Bruijn, J. Bussons, A. Camon, et al., in Space Telescopes and Instrumentation II: Ultraviolet to Gamma Ray. Edited by Turner, Martin J. L.; Hasinger, Günther. Proceedings of the SPIE, Volume 6266, pp. (2006). (2006).

[41] R. Kelley et al., PASJ 58 (2006).

[42] L. Amati, F. Frontera, M. Vietri, J. J. M. in't Zand, P. Soffitta, E. Costa, S. Del Sordo, E. Pian, L. Piro, L. A. Antonelli, et al., Science 290, 953 (2000), astro$\mathrm{ph} / 0012318$.

[43] F. Frontera, L. Amati, M. Vietri, J. J. M. i. Zand, E. Costa, M. Feroci, J. Heise, N. Masetti, L. Nicastro, M. Orlandini, et al., ApJ 550, L47 (2001), astro$\mathrm{ph} / 0102234$.

[44] R. F. Mushotzky, L. L. Cowie, A. J. Barger, and K. A. Arnaud, Nature 404, 459 (2000), astro-ph/0002313.

[45] M. A. Worsley et al., MNRAS 357, 1281 (2005), astro$\mathrm{ph} / 0412266$.

[46] E. Treister and C. M. Urry, ApJ 630, 115 (2005).

[47] J. M. Dickey and F. J. Lockman, Ann. Rev. Astron. Astrophys. 28, 215 (1990). 